\title{
EFEITO DE INSETICIDAS USADOS NA CULTURA DO MILHO SOBRE A CAPACIDADE DE PARASITISMO DE TRICHOGRAMMA PRETIOSUM RILEY, 1879 (HYMENOPTERA: TRICHOGRAMMATIDAE)
}

\author{
G.J. Stefanello Júnior ${ }^{*}$, A.D. Grützmacher², D.D. Grützmacher ${ }^{3}$, \\ G.O. Dalmazo ${ }^{4}$, M.D.F. Paschoal', W.R. Härter ${ }^{6}$
}

1Universidade Federal de Pelotas, Faculdade de Agronomia “Eliseu Maciel”, Departamento de Fitossanidade, CP 354, CEP 96010-900, Pelotas, RS, Brasil. E-mail: gstefanello@gmail.com

\section{RESUMO}

\begin{abstract}
Foi avaliado o efeito de dezesseis formulações comerciais de inseticidas registrados para a cultura do milho sobre a capacidade de parasitismo de Trichogramma pretiosum Riley, 1879 (Hymenoptera: Trichogrammatidae) em condições de laboratório (temperatura $25 \pm 1{ }^{\circ} \mathrm{C}$, umidade relativa $70 \pm 10 \%$; fotofase 14 horas e luminosidade 500 lux). Adultos do parasitóide foram colocados em contato com uma película seca dos inseticidas pulverizados sobre placas de vidro e a capacidade de parasitismo das fêmeas foi mensurada. A redução na capacidade de parasitismo dos tratamentos foi comparada com a testemunha (água destilada) e utilizada para classificar os inseticidas em quatro classes: 1, inócuo $(<30 \%)$; 2, levemente nocivo (30-79\%); 3, moderadamente nocivo $(80-99 \%)$ e 4 , nocivo $>99 \%)$. Os inseticidas (\% de ingrediente ativo na calda) Certero $(0,012)$ e Rimon 100 EC $(0,0075)$ foram inócuos; Fastac $100 S C(0,0025)$ e Match EC $(0,0075)$ foram levementenocivos; Tracer $(0,024)$ foi moderadamente nocivo; Arrivo 200 EC $(0,008)$, Decis 25 EC $(0,0025)$, Deltaphos EC $(0,00175$ deltametrina $+0,06125$ triazofós), Dipterex $500(0,5)$, Folidol $600(0,15)$, Hostathion 400 BR $(0,1)$, Karate Zeon 250 CS $(0,0125)$, Malathion 500 CESultox $(0,625)$, Sumithion 500 CE $(0,375)$, Turbo $(0,0025)$ e Vexter $(0,24)$ foram nocivos aos adultos de T. pretiosum no teste de toxicidade inicial em condições de laboratório.
\end{abstract}

PALAVRAS-CHAVE: Insecta, seletividade, parasitóide de ovos, pesticidas, Zea mays.

\section{ABSTRACT}

THE EFFECT OF INSECTICIDES USED IN CORN CROPS ON THE PARASITISM CAPACITY OF TRICHOGRAMMA PRETIOSUM RILEY, 1879 (HYMENOPTERA: TRICHOGRAMMATIDAE). The side-effects of sixteen commercial formulations of insecticides registered for corn crops were assessed for Trichogramma pretiosum Riley, 1879 (Hymenoptera: Trichogrammatidae) under laboratory conditions $\left(25 \pm 1^{\circ} \mathrm{C}\right.$ temperature, $70 \pm 10 \%$ relative humidity; $14 \mathrm{~h}$ photophase and brightness 500 lux). The adult parasitoids were submitted to fresh dry insecticide film applied on glass plates, and the capacity of parasitism of the females was evaluated. The reduction in capacity of parasitism for the respective treatments was compared with the control (distilled water) and used to classify the insecticides into four categories: 1, harmless $(<30 \%) ; 2$, slightly harmful $(30$ $79 \%) ; 3$, moderately harmful (80-99\%) and 4, harmful (>99\%). The pesticides (\% active ingredient in spray water) Certero (0.012) and Rimon 100 EC (0.0075) were harmless; Fastac 100 SC (0.0025) and Match EC (0.0075) were slightly harmful; Tracer (0.024) was moderately harmful; Arrivo 200 EC (0.008), Decis 25 EC (0.0025), Deltaphos EC (0.00175 deltamethrin + 0.06125 triazophos), Dipterex 500 (0.5), Folidol 600 (0.15), Hostathion 400 BR (0.1), Karate Zeon 250 CS (0.0125), Malathion 500 CE Sultox (0.0625), Sumithion 500 CE (0.375), Turbo (0.0025) and Vexter (0.24) were harmful to adults of T. pretiosum in the test of initial toxicity under laboratory conditions.

KEY WORDS: Insecta, selectivity, egg parasitoids, pesticides, Zea mays.

\footnotetext{
*Parte da Dissertação de Mestrado do primeiro autor, Programa de Pós-Graduação em Fitossanidade. Apoio: bolsa de estudos da Coordenação de Aperfeiçoamento de Pessoal de Nível Superior (CAPES).

${ }^{2}$ Dr., Prof., Pesquisador CNPq, Depto. de Fitossanidade-FAEM-UFPel, Pelotas, RS, Brasil.

${ }^{3}$ Dr., PRODOC-CAPES, Depto. de Fitossanidade-FAEM-UFPel, Pelotas, RS, Brasil.

${ }^{4}$ Acadêmico Agronomia, PIBIC CNPq, Depto. de Fitossanidade-FAEM-UFPel, Pelotas, RS, Brasil.

${ }^{5}$ Acadêmico Agronomia, PET, Depto. de Fitossanidade-FAEM-UFPel, Pelotas, RS, Brasil.

${ }^{6}$ Acadêmico Agronomia, BIC CNPq, Depto. de Fitossanidade-FAEM-UFPel, Pelotas, RS, Brasil.
} 


\section{INTRODUÇÃO}

A cultura do milho é hospedeira de vários insetospraga nas fases vegetativa e reprodutiva, destacandose a lagarta-do-cartucho Spodopterafrugiperda (Smith, 1797) (Lepidoptera: Noctuidae). O ataque de lagartas dessa espécie, que ocorrem desde a emergência das plantas atéo pendoamento eespigamento, pode reduzir a produção em até $34 \%$, sendo assim considerada a principal praga da cultura do milho no Brasil (CRUZ et al., 2002).

Dentre as alternativas que visam à redução populacional de $S$. frugiperda observa-se basicamente o controlequímico com ouso de inseticidas. Entretanto, neste método de controle existem problemas relacionados à tecnologia e momento de aplicação, assim favorecendo o surgimento de pragas de final de ciclo da cultura, como a lagarta-da-espiga Helicoverpa zea (Boddie, 1850) (Lepidoptera: Noctuidae), em função do desequilíbrio ocasionado pelos produtos químicos de amplo espectro (Borba et al., 2003).

Referente ao controle biológico na cultura do milho, várias espécies de inimigos naturais são citadas como importantes agentes de controle da lagarta-docartucho, tais como parasitóides, predadores, fungos, vírus, bactérias, nematóides e protozoários (CRUZ et al., 2002). No grupo dos parasitóides de ovos destacam-se aqueles pertencentes ao gênero Trichogramma, que apresenta como principal vantagem o controle das pragas ainda na fase de ovo, ou seja, antes destas causarem qualquer dano à cultura. A espécie Trichogramma pretiosum Riley, 1879 (Hymenoptera: Trichogrammatidae)caracteriza-se por sergeneralista, apresentando potencial para o controle biológico de lepidópteros-praga (BorBA et al., 2003) ou em associação com o controle químico a partir do uso de produtos seletivos (STEFANello Júnior et al., 2005).

No Brasil, a seletividade de agrotóxicos utilizados na cultura do milho a parasitóides tem sido pouco estudada (GonçaLves et al., 1997; Souza et al., 1998; Pratissolietal., 2004), principalmente para a espécieT. pretiosum (Pratissolietal., 2004). Observa-se, portanto, inexpressivo número de trabalhos a fim de avaliar a seletividade de agrotóxicos registrados para a cultura do milho ao parasitóide de ovos $T$. pretiosum, seja utilizando as metodologias padronizadas pelaIOBC/ WPRS (STEFANELLO JÚNIOR et al., 2005) ou outras distintas a essa (Pratissoli et al., 2004). Dessa maneira, verifica-se carência de dados de toxicidade de compostos a inimigos naturais e deficiência no suporte ao programadeManejoIntegradodePragas nacultura domilho, fato que prejudica a eficiência de outros métodos de supressão de insetos-praga como o controle biológico.

Dessa forma, o objetivo deste trabalho foi avaliar o efeito de inseticidas registrados para a cultura do milho sobre a capacidade de parasitismo de $T$. pretiosum em condições de laboratório, utilizando a metodologia padronizada pela "International Organization for Biological and Integrated Control of Noxious Animals and Plants(IOBC), WestPalaearctic Regional Section (WPRS)".

\section{MATERIAL E MÉTODOS}

Os trabalhos foram desenvolvidos nos Laboratórios de Controle Biológico e de Pesticidas da Universidade Federal de Pelotas. Os parasitóidesT.pretiosum foram coletados na cultura do milho, Município de Pelotas, RS, e multiplicados em laboratório utilizando-se ovos do hospedeiro alternativo Anagasta kuehniella (Zeller, 1879) (Lepidoptera: Pyralidae). Os testes de seletividade foram conduzidos em laboratório (temperatura $25 \pm 1^{\circ} \mathrm{C}$, umidade relativa $70 \pm$ $10 \%$ e fotofase 14 horas), expondo-se adultos (estágio mais sensível) dos parasitóides aos resíduos secos dos agrotóxicos (Tabela 1), conforme metodologia de Giolo et al. (2005).

A seletividade dos inseticidas ao inimigo natural foi avaliada utilizando-se a máxima dosagem registrada para a cultura do milho (Tabela 1). Os inseticidas foram diluídos em água destilada, considerando um volume de calda de $200 \mathrm{~L} \mathrm{ha}^{-1}$, e pulverizados em placas de vidro $(0,2 \mathrm{~cm}$ de espessura $\times 13 \mathrm{~cm} \times 13 \mathrm{~cm})$ que posteriormente foram utilizadas para confecção das gaiolas de exposição conforme HASSAN; Abdelgader (2001). A deposição de calda de 1,75 \pm $0,25 \mathrm{mg} \mathrm{cm}^{-2}$ foi aferida por pesagem em balança eletrônica de precisão.

Para testemunhas negativa e positiva, respectivamente, foram utilizadoságua destilada e um pesticida reconhecidamente nocivo (classe 4) pela IOBC (Lorsban $480 \mathrm{BR}$ ) (Tabela 1), também registrado para a cultura do milho.

Tubos de emergência (ampolas de vidro de $12 \mathrm{~cm}$ de comprimento $\times 2 \mathrm{~cm}$ de diâmetro na extremidade $x 0,7 \mathrm{~cm}$ na extremidade oposta) contendo adultos de T. pretiosum, com aproximadamente 24 horas de idade, foram conectados às gaiolas de exposição conforme Hassan; Abdelgader (2001). Seis horas após a desconexão dos tubos de emergência, cartões contendo 3 círculos de $1 \mathrm{~cm}$ de diâmetro com $450 \pm 50$ ovos de A. kuehniella inviabilizados e alimento (solução composta por $200 \mathrm{~g}$ de mel, $3 \mathrm{~g}$ de gelatina em pó sem sabor e $100 \mathrm{~mL}$ de água) foram oferecidos às 24 (três cartões), 48 (dois cartões) e 96 horas (um cartão) após pulverização para serem parasitados por T.pretiosum, totalizando 18 círculos, com aproximadamente 9.000 ovos por gaiola.

A avaliação da capacidade de parasitismo foi feita por até 144 horas (6 dias), sendo que, em seguida, as gaiolas foram desmontadas e os cartões foram preser- 
vados em placas de Petri $(9,0 \times 1,5 \mathrm{~cm})$, sendo armazenados nas mesmas condições do teste por mais três dias para que todos os ovos parasitados se tornassem escuros e pudessem ser contados.

Foram utilizadas quatro repetições para cada tratamento, sendo cada gaiola considerada uma unidade experimental no delineamento inteiramente casualizado. Cada produto foi enquadrado em classes de toxicidade conforme escala proposta pela IOBC/WPRS, baseada na redução de parasitismo de T. pretiosum quando comparada com a testemunha negativa.

Dessa forma, os inseticidas foram classificados em: 1, inócuo (<30\%); 2, levemente nocivo (30-79\%); 3 , moderadamente nocivo (80-99\%) e 4, nocivo (> $99 \%$ ), em função do produto comercial, conforme HASSAN et al. (2000), pois um mesmo ingrediente ativo poderá estar em distintas formulações comerciais e assim ocasionar impacto diferenciado sobre o inseto em teste. Análises estatísticas complementares foram realizadas com auxílio do programa estatístico SAS (SAs LeARning Edition, 2002).

\section{RESULTADOS E DISCUSSÃO}

O número médio de fêmeas de $T$. pretiosum no interior das gaiolas de exposição variou de 135,02 a 141,81 no experimento I; de 116,59 a 139,24 no experimento II; de 136,42 a 160,84 no experimento III e de 149,43 a 170,91 no experimento IV (Tabelas 2 e 3). De acordo com ZHANG; HASSAN (2000), valores entre 55 e 150 fêmeas de $T$. cacoeciae no interior das gaiolas de exposição não interferiram nos resultados obtidos pelos autores. Entretanto, HASSAN (1998a) relatou que o número de fêmeas no interior das gaiolas pode variar de 200 a 400 para a metodologia proposta pela IOBC/WPRS para T. cacoeciae.

$\mathrm{Na}$ testemunha negativa, os valores obtidos de ovos parasitados por fêmea foram de 35,07; 27,74; 27,17 e 21,75 nos experimentos I, II, III e IV, respectivamente (Tabelas 2 e 3). Para a espécie T. cacoeciae, os membros da IOBC preconizam um mínimo de 15 ovos parasitados por fêmea (HASSAN et al., 2000). Para T. pretiosum ainda não há um número mínimo estabelecido para validação de testes de seletividade. Entretanto, MACEDA et al. (2003) estudaram os efeitos de temperatura sobre o desenvolvimento desta espécie de parasitóide em ovos de A. kuehniella e observaram que, em temperatura de $25^{\circ} \mathrm{C}$, as fêmeas parasitaram em média 5,74 ovos por dia. De acordo com os resultados de parasitismo de T. pretiosum observados na maioria dos experimentos deste trabalho, em seis dias, verificam-se valores similares aos encontrados por esses autores.

O parasitismo das fêmeas de T. pretiosum variou significativamente entre os inseticidas testados nos experimentos I a IV, variando de zero a 35,07 ovos por fêmea. As reduções no parasitismo variaram de 1,5 a $100 \%$, predominando os inseticidas classificados como nocivos (classe 4 ) e que representaram $68,75 \%$ dos inseticidas testados (Tabelas 2 e 3).

Tabela 1 - Inseticidas avaliados nos testes de seletividade a adultos de Trichogramma pretiosum.

\begin{tabular}{lllll}
\hline Produto Comercial & Ingrediente Ativo & Grupo Químico & DC $^{1}$ & C.i.a. $(\%)^{2}$ \\
\hline Arrivo 200 EC & cipermetrina & Piretróide & 0,08 & 0,008 \\
Certero & triflumurom & Benzoiluréia & 0,05 & 0,012 \\
Decis 25 EC & deltametrina & Piretróide & 0,20 & 0,0025 \\
Deltaphos EC & deltametrina + triazofós & Piretróide + Organofosforado & 0,35 & $0,00175+0,06125$ \\
Dipterex 500 & triclorfom & Organofosforado & 2,00 & 0,5 \\
Fastac 100 SC & alfa-cipermetrina & Piretróide & 0,05 & 0,0025 \\
Folidol 600 & parationa-metílica & Organofosforado & 0,50 & 0,15 \\
Hostathion 400 BR & triazofós & Organofosforado & 0,50 & 0,1 \\
Karate Zeon 250 CS & lambda-cialotrina & Piretróide & 0,10 & 0,0125 \\
Malathion 500 CE Sultox & malationa & Organofosforado & 2,50 & 0,625 \\
Match EC & lufenurom & Benzoiluréia & 0,30 & 0,0075 \\
Rimon 100 EC & novalurom & Benzoiluréia & 0,15 & 0,0075 \\
Sumithion 500 CE & fenitrotiona & Organofosforado & 1,50 & 0,375 \\
Tracer & espinosade & Espinosinas & 0,10 & 0,024 \\
Turbo & beta-ciflutrina & Piretróide & 0,10 & 0,0025 \\
Vexter & clorpirifós & Organofosforado & 1,00 & 0,24 \\
Lorsban 480 BR & clorpirifós & Organofosforado & 1,00 & 0,24 \\
\hline
\end{tabular}

${ }^{1} \mathrm{DC}=$ Dosagem de campo (L ha- ${ }^{-1}$ do produto comercial) considerando um volume de calda de $200 \mathrm{~L} \mathrm{ha}^{-1}$;

${ }^{2}$ C.i.a. = Concentração do ingrediente ativo na calda utilizada no teste de seletividade;

${ }^{3}$ Testemunha positiva, inseticida reconhecidamente nocivo pela IOBC/WPRS. 
Tabela 2-Efeitos de inseticidas registrados para a cultura do milho sobre o número médio de ovos parasitados por fêmea, redução (\%) na capacidade de parasitismo de Trichogramma pretiosum e classificação de toxicidade dos inseticidas. Experimentos I e II. Temperatura $25 \pm 1^{\circ} \mathrm{C}$; umidade relativa $70 \pm 10 \%$; fotofase 14 horas. Pelotas, RS. 2006.

\begin{tabular}{|c|c|c|c|c|}
\hline Produto comercial/ingrediente ativo & Fêmeas por gaiola & Ovos por fêmea* & $\mathrm{RP}^{* *}(\%)$ & Classes IOBC ${ }^{* * *}$ \\
\hline \multicolumn{5}{|l|}{ Experimento I } \\
\hline Água destilada/testemunha negativa & 141,14 & 35,07 a & -- & - \\
\hline Dipterex 500/triclorfom & 141,81 & $0,00 \mathrm{~b}$ & 100 & 4 \\
\hline Malathion 500 CE Sultox/malationa & 140,04 & $0,00 \mathrm{~b}$ & 100 & 4 \\
\hline Sumithion $500 \mathrm{CE} /$ fenitrotiona & 136,83 & $0,00 \mathrm{~b}$ & 100 & 4 \\
\hline Vexter/clorpirifós & 135,02 & $0,00 \mathrm{~b}$ & 100 & 4 \\
\hline Lorsban 480 BR/clorpirifós ${ }^{* * * *}$ & 135,69 & $0,00 \mathrm{~b}$ & 100 & 4 \\
\hline \multicolumn{5}{|l|}{ Experimento II } \\
\hline Água destilada/testemunha negativa & 139,24 & $27,74 \mathrm{a}$ & -- & - \\
\hline Certero/triflumurom & 131,89 & $27,32 \mathrm{ab}$ & 1,50 & 1 \\
\hline Rimon 100 EC/novalurom & 116,59 & $22,84 \mathrm{abc}$ & 17,66 & 1 \\
\hline Match EC/lufenurom & 136,39 & $14,42 \mathrm{~cd}$ & 48,02 & 2 \\
\hline Tracer/espinosade & 134,26 & 1,32 de & 95,26 & 3 \\
\hline Lorsban 480 BR/clorpirifós ${ }^{* * * *}$ & 136,94 & 0,00 & 100 & 4 \\
\hline
\end{tabular}

*Médias seguidas por letras idênticas não diferem significativamente pelo teste (Kruskal-Wallis) Bonferroni ( $p>0,05)$; Experimento I $(K=22,76, p=0,0004)$; Experimento II $(K=19,54, p=0,0015)$. Os resultados expressam a média de quatro repetições.

** RP = Redução na capacidade de parasitismo dos tratamentos com inseticidas comparados com a testemunha negativa (água destilada);

***Classes da IOBC para teste de toxicidade inicial sobre adultos: 1 = inócuo $(<30 \%), 2$ = levemente nocivo ( $30-79 \%), 3$ $=$ moderadamente nocivo $(80-99 \%)$ e $4=$ nocivo $(>99 \%)$;

****Testemunha positiva, inseticida reconhecidamente nocivo pela IOBC/WPRS.

Os inseticidas (\% de ingrediente ativo na calda) do grupo químico dos organofosforados Dipterex 500 $(0,5)$, Malathion 500 CE Sultox $(0,625)$, Folidol 600 $(0,15)$, Hostathion 400 BR $(0,1)$, Sumithion $500 \mathrm{CE}$ $(0,375)$ e Vexter $(0,24)$ causaram redução de $100,00 \%$ na capacidade de parasitismo, sendo classificados como nocivos (classe 4) a adultos de $T$. pretiosum (Tabelas 2 e 3). DeltaphosEC $(0,00175$ deltametrina + 0,06125 triazofós), Arrivo $200 \mathrm{EC}(0,008)$, Decis $25 \mathrm{EC}$ $(0,0025)$, Karate Zeon 250 CS $(0,0125)$ e Turbo $(0,0025)$ também causaram redução de $100,00 \%$ na capacidade de parasitismo, sendo classificados como nocivos (classe 4). O inseticida Fastac 100 SC $(0,0025)$ apresentou $46,77 \%$ de redução no parasitismo e foi classificado como levemente nocivo (classe 2 ) a adultos de T. pretiosum (Tabela 3 ).

Resultados semelhantes para Dipterex 500 também foram encontrados por Giolo et al. (2005) e GRÜTZMACHER et al. (2004), ao trabalharem com inseticidas registrados para a cultura do pessegueiro, que classificaram o inseticida como nocivo (classe 4) a adultos $T$. pretiosum e $T$. cacoeciae, respectivamente, embora tenham utilizado uma calda com menor porcentagem de ingrediente ativo $(0,15)$ quando comparada ao presente trabalho $(0,5)$. O inseticida Malathion 1000 CE $(0,1)$ também foi classificado como nocivo (classe 4) em testes de seletividade que avaliaram o parasitismo de adultos de T. pretiosum por MANZONI et al. (2006) ao testarem produtos registrados para a cultura da macieira. Sumithion $500 \mathrm{CE}$ foi classificado como nocivo (classe 4) a adultos de $T$. pretiosum (GRÜTZMACHERet al., 2005) em trabalhos utilizando agrotóxicos registrados para a cultura do pessegueiro na concentração de $0,075 \%$ de ingrediente ativo na calda, cinco vezes menos se comparado ao presente trabalho $(0,375)$ (Tabela 1$)$.

Vexter $(0,096)$ foi classificado como nocivo (classe 4 ) a adultos de $T$. pretiosum por STEFANELLOJÚNIOR et al. (2005), pois reduziu em $100 \%$ o parasitismo quando os autores testaram agrotóxicos registrados para a cultura do milho, embora tenham utilizado $60 \%$ a menos de ingrediente ativo $(0,096)$ se comparado ao presente trabalho $(0,24)$. Lorsban $480 \mathrm{BR}$ $(0,072)$ também foi classificado como nocivo (classe 4) a adultos de T. pretiosum por GRÜTZMACHER et al. (2005) ao testar produtos registrados para a cultura da macieira.

A mesma classificação também foi obtida por HASSAN (1998b) ao testar Dursban Spritzp. (0,25\% do produto comercial), contendo clorpirifós como ingrediente ativo, a adultos de T. cacoeciae. 
Tabela 3 - Efeitos de inseticidas registrados para a cultura do milho sobre o número médio de ovos parasitados por fêmea, redução (\%) na capacidade de parasitismo de Trichogramma pretiosum e classificação de toxicidade dos inseticidas. Experimentos III e IV. Temperatura $25 \pm 1^{\circ}$ C; umidade relativa $70 \pm 10 \%$; fotofase 14 horas. Pelotas, RS. 2006.

\begin{tabular}{|c|c|c|c|c|}
\hline Produto comercial/ingrediente ativo & Fêmeas por gaiola & Ovos por fêmea* & $\mathrm{RP}^{* *}(\%)$ & Classes IOBC ${ }^{* * *}$ \\
\hline \multicolumn{5}{|l|}{ Experimento III } \\
\hline Água destilada/testemunha negativa & 160,84 & 27,17 a & -- & - \\
\hline Fastac 100 SC/alfa-cipermetrina & 159,30 & $14,46 \mathrm{~b}$ & 46,77 & 2 \\
\hline Arrivo 200 EC/cipermetrina & 150,15 & $0,00 \quad \mathrm{c}$ & 100 & 4 \\
\hline Decis $25 \mathrm{EC} /$ deltametrina & 136,42 & 0,00 & 100 & 4 \\
\hline Karate Zeon 250 CS/lambda-cialotrina & 137,56 & 0,00 & 100 & 4 \\
\hline Lorsban 480 BR/clorpirifós ${ }^{* * * *}$ & 155,40 & 0,00 & 100 & 4 \\
\hline \multicolumn{5}{|l|}{ Experimento IV } \\
\hline Água destilada/testemunha negativa & 170,91 & $21,75 \mathrm{a}$ & -- & - \\
\hline Deltaphos EC/deltametrina + triazofós & 163,56 & $0,00 \mathrm{~b}$ & 100 & 4 \\
\hline Folidol 600/parationa-metílica & 149,43 & $0,00 \mathrm{~b}$ & 100 & 4 \\
\hline Hostathion 400 BR/triazofós & 165,53 & $0,00 \mathrm{~b}$ & 100 & 4 \\
\hline Turbo/beta-ciflutrina & 161,92 & $0,00 \quad b$ & 100 & 4 \\
\hline Lorsban 480 BR/clorpirifós**** & 164,19 & $0,00 \mathrm{~b}$ & 100 & 4 \\
\hline
\end{tabular}

*Médias seguidas por letras idênticas não diferem significativamente pelo teste (Kruskal-Wallis) Bonferroni ( $p>0,05)$; Experimento III $(K=22,716, p=0,0004)$; Experimento IV $(K=22,7629, p=0,0004)$. Os resultados expressam a média de quatro repetições.

${ }^{* *} \mathrm{RP}=$ Redução na capacidade de parasitismo dos tratamentos com inseticidas comparados com a testemunha negativa (água destilada);

***Classes da IOBC para teste de toxicidade inicial sobre adultos: $1=$ inócuo $(<30 \%), 2=$ levemente nocivo $(30-79 \%)$, $3=$ moderadamente nocivo $(80-99 \%)$ e 4 = nocivo $(>99 \%)$;

****Testemunha positiva, inseticida reconhecidamente nocivo pela IOBC/WPRS.

O ingrediente ativo triazofós, presente nos inseticidas Hostathion 400 BRe Deltaphos EC (deltametrina + triazofós), foi nocivo (classe 4) a T. pretiosum. A mesma classificação foi obtida porHASSAN et al.(1988) aos adultos de T. cacoeciae, ao testarem o inseticida Hostathion numa concentração de $0,24 \%$ do produto comercial. O ingrediente ativo deltametrina, presente na formulação comercial do inseticida Decis, foi classificado como nocivo (classe 4 ) aos adultos de $T$. cacoeciae por HASSAN et al. (1987) ao testarem o inseticida na concentração de $0,06 \%$ do produto comercial. Os inseticidas Karate 50 EC (0,0038\% lambdacialotrina) e Baythroid 50 EC (0,0025\% ciflutrina) (STERK et al., 1999) foram classificados como nocivos (classe 4) aos adultos de T. cacoeciae.

O produto Fastac 100 SC $(0,0025)$ apresentou a mesma concentração de ingrediente ativo que Decis 25 EC $(0,0025)$ e Turbo $(0,0025)$ na calda utilizada na pulverização, porém verificou-se seletividade diferenciada a adultos T. pretiosum e foi considerado levemente nocivo (classe 2). Uma hipótese a esse resultado adverso, em relação aos demais piretróides testados, pode ser em função dos diferentes ingredientes ativos presentes nos produtos comerciais e que pode ter refletido numa toxicidade diferenciada do produto.
Os inseticidas do grupo químico das benzoiluréias, Certero $(0,012)$ e Rimon 100 EC $(0,0075)$, causaram redução de 1,50 e $17,66 \%$ na capacidade de parasitismo de $T$. pretiosum, sendo classificados como inócuos (classe 1) (Tabela 2). Resultados semelhantes foram obtidos por CARVALHO et al. (2005) ao testarem inseticidas utilizados na tomaticultura, e classificaram o inseticida Certero 480 SC como inócuo (classe 1) a fêmeas de T. pretiosum. GRÜTZMACHERet al. (2005) classificaram Certero $(0,0048)$ e Rimon 100 EC $(0,005)$ como inócuos (classe 1) a adultos de T. pretiosum, ao testarem os agrotóxicos registrados para a cultura da macieira.

O inseticida do grupo químico das benzoiluréias Match EC $(0,0075)$ reduziu em $48,02 \%$ a capacidade de parasitismo de $T$. pretiosum, sendo classificado como levemente nocivo (classe 2) (Tabela 2). Resultados semelhantes foram obtidos porGRÜTZMACHERet al. (2005) ao testarem Match EC $(0,005)$, classificando o inseticida como levemente nocivo (classe 2) a adultos de T.pretiosum; entretanto, ao utilizarem $0,004 \%$ de ingrediente ativo na calda, os autores classificaram o inseticida como inócuo (classe 1).

Match 50 EC, entretanto, foi considerado como seletivo a T. pretiosum por PRATISSOLI et al. (2004), quando os autores ofertaram ovos de $S$. frugiperda 
contaminados com calda inseticida na concentração de $15,0 \mathrm{~g}$ i.a. $\mathrm{L}^{-1}$.

O inseticida do grupo químico das espinosinas Tracer $(0,024)$ reduziu em $95,26 \%$ a capacidade de parasitismo de fêmeas de T. pretiosum, sendo classificado como moderadamente nocivo (classe 3) (Tabela 2). CAÑETE (2005) avaliou a seletividade de inseticidas utilizados em cultura da soja ao ofertar ovos de Anticarsia gemmatalis Hübner, 1818 (Lepidoptera: Noctuidae) tratados com Tracer $\left(12 \mathrm{~g} \mathrm{ha}^{-1}\right.$ de ingrediente ativo espinosade) para T. pretiosume obteve $100 \%$ de mortalidade das fêmeas do parasitóide.

Observa-se que os inseticidas organofosforados, piretróides e espinosinas apresentam elevada toxicidadea adultos devárias espécies de parasitóides. Segundo Foerster(2002), a possibilidade de obtenção de seletividade fisiológica com inseticidas neurotóxicos é reduzida em virtude da similaridade no processo de transmissão dos impulsos nervosos não apenas entre as diferentes ordens de insetos, mas também entre os vários filos animais.

Os testes de toxicidade inicial em laboratório submetem os insetos-teste à máxima exposição aos resíduos dos agrotóxicos e constituem a primeira etapa da seqüência de testes preconizada pela IOBC/WPRS (Hassan et al., 2000; Hassan; Abdelgader, 2001). Nesse sentido, os inseticidas inócuos (classe 1) podem ser utilizados juntamente com adultos de T. pretiosum, porém os nocivos (classes 2, 3, e 4) deverão passar para os testes em laboratório sobre imaturos do parasitóide e em casa-de-vegetação, para avaliar a toxicidade sobreas fases embrionárias do parasitóide e a persistência biológica, respectivamente.

\section{CONCLUSÕES}

Os inseticidas (\% de ingrediente ativo na calda) Certero $(0,012)$ e Rimon 100 EC $(0,0075)$ são inócuos (classe 1); Fastac 100 SC $(0,0025)$ e Match EC $(0,0075)$ são levemente nocivos (classe 2); Tracer $(0,024)$ é moderadamente nocivo (classe 3); Arrivo 200 EC $(0,008)$, Decis 25 EC $(0,0025)$, Deltaphos EC $(0,00175$ deltametrina $+0,06125$ triazofós), Dipterex $500(0,5)$, Folidol $600(0,15)$, Hostathion 400 BR $(0,1)$, Karate Zeon 250CS (0,0125), Malathion500 CESultox (0,625), Sumithion 500 CE $(0,375)$, Turbo $(0,0025)$ e Vexter $(0,24)$ são nocivos (classe 4$)$, pois reduzem a capacidade de parasitismo de T. pretiosum.

\section{REFERÊNCIAS}

BASTOS, C.S.; ALMEIDA, R.P. DE; SUINAGA, F.A. Selectivity of pesticides used on cotton (Gossypium hirsutum) to Trichogramma pretiosum reared on two laboratory-reared hosts. Pest Management Science, v.62, n.1, p.91-98, 2005.
BORBA, R. DA S.; BUSATO, G.R.; FORESTI, J.; GIOLO, F.P.; GARCIA, M.S.; GRÜTZMACHER, A.D. Avaliação de espécies ou linhagens de Trichogramma para o parasitismo de Spodoptera frugiperda em condições de laboratório. In: REUNIÃO TÉCNICA ANUAL DO MILHO, SORGO E FEIJÃO, 47., 30., 35., 2002, Porto Alegre, RS. Anais. Porto Alegre: 2003. Cd-rom.

CAÑETE, C.L. Seletividade de inseticidas a espécies de Trichogramma (Hymenoptera: Trichogrammatidae). 2005. 106f. Tese (Doutorado em Ciências - Área de Zoologia) - Setor de Ciências Biológicas, Universidade Federal do Paraná, Curitiba, 2005.

CARVALHO, G.A.; REZENDE, D.T.; MOURA, A.P.; MOSCARDINI, V.F.; LASMAR, O.; SOUZA, J.R. Selectivity of flubendiamide, a new inseticide used to control tomato pests in Brazil to Trichogramma pretiosum Riley (Hym., Trichogrammatidae). Egg Parasitoid News, n.17, 2005. Disponível em: <http:/ / www.bba.de/eggpara/ eggp.htm>.Acesso em: 17 nov. 2006.

CRUZ, I.; VIANA, P.A.; WAQUIL, J.M. Cultivo do milho, pragas da fase vegetativa e reprodutiva. Sete Lagoas: Embrapa Milho e Sorgo, 2002. 8p. (Comunicado Técnico, 49).

FOERSTER, L.A. Seletividade de inseticidas a predadores e parasitóides. In: PARRA, J.R.P.; BOTELHO, P.S.M. CORREAA-FERREIRA, B.S.; BENTO, J.M.S. (Ed.). Controle biológico no Brasil: parasitóides e predadores. São Paulo: Manole, 2002. cap.6, p.95-114.

GIOLO, F.P.; GRÜTZMACHER, A.D.; MANZONI, C.G.; FACHINELLO, J.C.; NÖRNBERG, S.D.; STEFANELLO JÚNIOR, G.J. Seletividade de agrotóxicos indicados na produção integrada de pêssego a Trichogramma pretiosum Riley, 1879 (Hymenoptera:

Trichogrammatidae). Revista Brasileira de Fruticultura, v.27, n.2, p.222-225, 2005.

GONÇALVES, E.P.; CRUZ, I.; FIGUEIREDO, M.L.C.; CIOCIOLA JUNIOR, A.I. Efeito do produto "spinosad" sobre lagartas de Spodoptera frugiperda Smith e sobre seus inimigos naturais, o predador Doru luteipes Scudder e o parasitóide Campoletis flavicincta Ashmead. In: CONGRESSO BRASILEIRO DE ENTOMOLOGIA, 16., 1997, Salvador, BA. Resumos. Salvador: 1997. p.177.

GRÜTZMACHER, A.D.; ZIMMERMANN, O.; YOUSEF, A.; HASSAN, S.A. The side-effects of pesticides used in integrated production of peaches in Brazil on the egg parasitoid Trichogramma cacoeciae Marchal (Hym.; Trichogrammatidae). Journal of Applied Entomology, v.128, n.6, v.377-383, 2004.

GRÜTZMACHER, A.D.; GIOLO, F.P.; MANZONI, C.G.; HÄRTER, W.R.; NÖRNBERG, S.D. The side-effects of insect growth regulators used in apple orchards on adults of 
Trichogramma pretiosum Riley (Hym., Trichogrammatidae). Egg Parasitoid News, Braunschweig, n.17, 2005. Disponível em: <http://www.bba.de/eggpara/eggp.htm>. Acesso em: 17 nov. 2006.

HASSAN, S. Guideline for the evaluation of side effects of plant protection products on Trichogramma cacoeciae Marchal (Hym., Trichogrammatidae). IOBCMPRS, Bulletin, v.21. n.6, p.119-128, 1998a.

HASSAN, S.A. The side effects os 161 pesticides on the egg parasitoid Trichogramma cacoeciae Marchal (Hymenoptera, Trichogrammatidae). Egg Parasitoids, p.63-76, March, 1998b.

HASSAN, S.; ABDELGADER, H. A sequential testing program to assess the side effects of pesticides on Trichogramma cacoeciae Marchal (Hym.,

Trichogrammatidae). Pesticides and Beneficial Organisms. IOBC/WPRS Bulletin, Darmstadt, v.24, n.4, p.71-81, 2001.

HASSAN, S.A.; ALBERT, R.; BIGLER, F.; BLAISINGER, P.; BOGENSCHÜTZ, H.; BOLLER, E.; BRUN, J.; CHIVERTON, P.; EDWARDS, P.; ENGLERT, W.D.; HUANG, P.; INGLESFIELD, C.; NATON, E.; OOMEN, P.A.; OVERMEER, P.J.; RIECKMANN, W.; SAMSOEPETERSEN, L.; STÄUBLI, A.; TUSET, J.J.; VIGGIANI, G.; VANWETSWINKEL, G. Results of the third joint pesticide testing programme by the IOBC/WPRSWorking Group "Pesticides and Beneficial Organisms". Journal of Applied Entomology, v.103, p.92107, 1987.

HASSAN, S.A.; BIGLER, F.; BOGENSCHÜTZ, H.; BOLLER, E.; BRUN, J.; CHIVERTON, P.; EDWARDS, P.; MANSOUR, F.; NATON, E.; OOMEN, P.A.; OVERMEER, W.P.J.; POLGAR, L.; RIECKMANN, W.; SAMSOE-PETERSEN, L.; STÄUBLI, A.; STERK, G.; TAVARES, K.; TUSET, J.J.; VIGGIANI, G.; VIVAS, A.C. Results of the fourth joint pesticide testing programme carried out by the IOBC/WPRS-Working Group "Pesticides and Beneficial Organisms. Journal of Applied Entomology, v.105, p.321-329, 1988.

HASSAN, S.A.; HALSALL, N.; GRAY, A.P.; KUEHNER, C.; MOLL, M.; BAKKER, F.M.; ROEMBKE, J.; YOUSEF,

A.; NASR, F.; ABDELGADER, H. A laboratory method to evaluate the side effects of plant protection products on Trichogramma cacoeciae Marchal (Hym.,

Trichogrammatidae), In: CANDOLFI, M.P.; BLUMEL, S.; FORSTER, R.; BAKKER, F.M.; GRIMM, C.; HASSAN, S.A.; HEIMBACH, U.; MEAD-BRIGGS, M.A.; REBER, B.; SCHMUCK, R.; VOGT, H. (Ed.). Guidelines to evaluate side-effects of plant protection products to non-target arthropods. Gent: IOBC/WPRS, 2000. p.107-119.

MACEDA, A.; HOHMANN, C.L.; SANTOS, H.R. Temperature effects on Trichogramma pretiosum Riley and Trichogrammatoidea annulata De Santis. Brazilian Archives of Biology and Technology, v.46, n.1, p.27-32, 2003.
MANZONI, C.G.; GRÜTZMACHER, A.D.; GIOLO, F.P.; HÄRTER, W.R.; MÜLLER, C. The side-effects of plant protection products used in integrated production of apples on adults of Trichogramma atopovirilia Oatman \& Platner (Hym., Trichogrammatidae). Egg Parasitoid News, Braunschweig, n.17, 2005. Disponível em: <www.bba.de/eggpara/eggp.htm>.Acesso em: 17 nov. 2006.

MANZONI, C.G.; GRÜTZMACHER, A.D.; GIOLO, F.P.; LIMA, C.A.B. de.; NÖRNBERG, S.D.; HARTER, W.DA.R.; MÜLLER, C. Seletividade de agrotóxicos recomendados na produção integrada de maçã a Trichogramma pretiosum Riley, 1879 (Hym.: Trichogrammatidae) em condições de laboratório. Revista Brasileira de Fruticultura, v.28, n.2, p.254-257, 2006.

PRATISSOLI, D.; THULER, R.T.; PEREIRA, F.F.; REIS, E.F. DOS; FERREIRA, A.T. Ação transovariana de lufenuron $(50 \mathrm{G} / \mathrm{L})$ sobre adultos de Spodoptera frugiperda (J.E. Smith) (Lepidoptera: Noctuidae) e seu efeito sobre o parasitóide de ovos Trichogramma pretiosum Riley (Hymenoptera: Trichogrammatidae). Ciência e Agrotecnologia, v.28, n.1, p.9-14, 2004.

SAS Learning Edition. Getting Started with the SAS Learning Edition. Cary, North Carolina: SAS Institute Inc. 2002.

SOUZA, A.C.; SILVA, A.S.; CRUZ, I.; FIGUEIREDO, M.L.C.; CARVALHO, E.R. Seletividade do inseticida Danimen (fenpropathrin, $300 \mathrm{~g} / \mathrm{L}$ ) para Campoletis flavicincta e Doru luteipes, inimigos naturais de Spodoptera frugiperda. In: CONGRESSO BRASILEIRO DE ENTOMOLOGIA, 17., 1998, Rio de Janeiro, RJ. Resumos Livro 1. Rio de Janeiro: 1998, p.83.

STEFANELLO JÚNIOR, G.J.; GRÜTZMACHER, A.D.; GRÜTZMACHER, D.D.; GIOLO, F.P.; DALMAZO, G.O. The side-effects of insecticides used in corn production in Brazil on adults of Trichogramma pretiosum Riley (Hym., Trichogrammatidae). Egg Parasitoid News, Braunschweig, n.17, 2005. Disponível em: <http:/ / www.bba.de/eggpara/eggp.htm>. Acesso em: 17 nov. 2006.

STERK, G.; HASSAN, S.A.; BAILLOD, M.; BAKKER, F.; BIGLER, F.; BLÜMEL, S.; BOGENSCHÜTZ, H.; BOLLER, E.; BROMAND, B.; BRUN, J.; CALIS, J.N.M.; COREMANS-PELSENEER, J.; DUSO, C.; GARRIDO, A.; GROVE, A.; HEIMBACH, U.; HOKKANEN, H.; JACAS, J.; LEWIS, G.; MORETH, L.; POLGAR, L.; ROVERSTI, L.; SAMSOE-PETERSEN, L.;

SAUPHANOR, B.; SCHAUB, L.; STÄUBLI, A.; TUSET, J.J.; VAINIO, A.; VAN DE VEIRE, M.; VIGGIANI, G.; VIÑUELA, E.; VOGT, H. Results of the seventh joint pesticide testing programme carried out by the IOBC/WPRS-Working Group 'Pesticides and Beneficial Organisms'. BioControl, v.44, p.99-117, 1999. 
YOUSSEF, A.I.; NASR, F.N.; STEFANOS, S.S.;

ELKHAIR, S.S.A.; SHEHATA, W.A.; AGAMY, E.; HERZ, A.; HASSAN, S.A. The side-effects of plant protection products used in olive cultivation on the hymenopterous egg parasitoid Trichogramma cacoeciae Marchal. Journal of Applied Entomology, v.128, n.9/10, p.593-599, 2004.
ZHANG, W.; HASSAN, S.A. Rationalising the standard method to test the side-effects of pesticides on Trichogramma cacoeciae, reducing the number of parasitoids tested. IOBCMPRS Bulletin, v.23, p.49-53, 2000.

Recebido em 9/2/07

Aceito em 16/5/08 